\title{
Change of Young's Modulus with Increasing Applied Tensile Strain in Open Cell Nickel and Copper Foams
}

\author{
Shojiro Ochiai ${ }^{1}$, Satoshi Nakano ${ }^{1, *}$, Yuya Fukazawa ${ }^{1, *}$, Mohamed Shehata Aly ${ }^{1}$, \\ Hiroshi Okuda $^{1}$, Komei Kato ${ }^{2}$, Takeshi Isobe ${ }^{3}$, Koichi Kita ${ }^{4}$ and Keiichi Honma ${ }^{3}$ \\ ${ }^{1}$ Department of Materials Science and Engineering, Kyoto University, Kyoto 606-8501, Japan \\ ${ }^{2}$ Mitsubishi Materials Corporation, Saitama 330-8508, Japan \\ ${ }^{3}$ Non-ferrous Alloys Research \& Technology Laboratory, Mitsubishi Materials Corporation, Kitamoto 364-0023, Japan \\ ${ }^{4}$ Central Research Institute, Mitsubishi Materials Corporation, Kitamoto 364-0022, Japan
}

The change of Young's modulus with increasing applied tensile strain in the open cell nickel and copper foams, fabricated by the slurry foaming process at Mitsubishi Materials Corporation, Japan, was investigated experimentally and analytically. Stress-strain curves were measured at room temperature, in which the loading-unloading-reloading process was performed on the sample at various applied strains. For measurement of the strain of the sample itself accurately, the non-contact extensometer was employed. The Young's modulus values at various applied tensile strains were estimated from the slope of the elastic region in the stress-strain curve in the unloading process. It was revealed that two competitive factors affect on the Young' modulus value. One is the irreversible morphology-change of cells arising from the plastic deformation of the metal as a results of which, the cells are elongated and struts are straight forwarded in the tensile direction. This factor acts to raise the Young's modulus with increasing applied strain. Another factor is the failure of struts, which tends to reduce the Young's modulus with increasing applied strain, especially beyond the strain where the struts are failed successively. Accordingly, due to the competition of the former and latter factors, the Young's modulus increases, reaching maximum and then decreases with increasing applied strain in both of the foams. The increase in Young's modulus with increasing applied strain due to the change of geometry of struts was confirmed by the experimental test of micro-samples composed of several struts and nodes and by the simulation based on the finite element analysis using a model micro-sample. [doi:10.2320/matertrans.M2009384]

(Received November 19, 2009; Accepted February 9, 2010; Published March 25, 2010)

Keywords: nickel foam, copper foam, deformation, failure, Young's modulus

\section{Introduction}

The metal foams have been produced by gas-injection method, ${ }^{1,2)}$ directional solidification method, ${ }^{3-5)}$ precursor method, ${ }^{6-8)}$ slurry foaming method ${ }^{9)}$ and so on. ${ }^{10-13)}$ Among them, the metal foam produced by the slurry foaming method has a feature of very high porosity fraction $(90 \sim 97 \%)$ in the open cell structure. ${ }^{9)}$ In the preceding work, ${ }^{14)}$ the deformation and failure behavior of nickel and copper open cell foams with porosity volume fraction $\approx 96 \%$ and pore size $\approx 600 \mu \mathrm{m}$ on average, fabricated by the slurry foaming method $^{9)}$ at Mitsubishi Materials Corporation, was studied. The following phenomena were found.

(1) The change of the morphology of the struts and cells is caused by the applied strain-induced plastic deformation, according to which the struts and cells are elongated and are straight forwarded in the tensile direction.

(2) In the early stage, the number of failed struts increases with increasing applied strain, where the failed struts are distributed spatially. On the other hand, in the later stage, the failed struts cause the failure of the neighboring struts one after another nearly in a limited cross-section.

In the bulk materials, the externally applied stress is borne by the whole body of the sample, and the Young's modulus is the material constant, which never varies under deformation. On the other hand, as stated above, in the present foams with high porosity ( $\approx 96 \%$ ), the stress is carried by the originally curved cells and struts. The morphology of the stress carrying

*Graduate Student, Kyoto University metal cells and struts is changed and also the failure of them is caused by the applied strain. It is expected that the Young's modulus varies with increasing applied strain. The aim of the present work is to estimate the Young's modulus of the foam and to discuss the influence of the change of the morphology of the cells and struts and failure of the struts on the change of the Young's modulus.

\section{Experimental and Analytical Procedure}

Open cell nickel and copper foam-plates with average porosity volume fraction $\approx 96 \%$ and pore size $\approx 600 \mu \mathrm{m}$, fabricated by the slurry foaming method at Mitsubishi Materials Corporation, were used as similarly as in our preceding paper. ${ }^{14)}$ The thicknesses of the nickel and copper foam plates were 2.0 and $1.5 \mathrm{~mm}$, respectively.

Tensile test was carried out at room temperature with a tensile machine (AUTOGRAPH-AG 50kNG, Shimadzu Corp). The width and gage length of tensile samples were 10 and $50 \mathrm{~mm}$, respectively. The test was conducted at a constant crosshead speed of $8.3 \times 10^{-6} \mathrm{~m} / \mathrm{s}$. The strain measurement of samples was carried out with a non-contact extensometer (DVW-200 Shimadzu), with which the strain of the samples itself was measured accurately without giving damage and constraint to the samples. Details of the sample specification and test procedure are shown in our preceding work. ${ }^{14)}$

In the measurement of stress-strain curves of the foams for estimation of Young's modulus, the sample was loaded up to a prescribed strain, and was unloaded in a small strain 
range, and then was reloaded to the next prescribed strain. Such a loading-unloading-reloading process was given to the sample repeatedly. The Young's modulus of the foam samples was estimated using the elastic deformation region that appears in the stress-strain curve upon unloading.

As shown later, the change of the Young's modulus of foams with increasing applied strain is attributed to the morphology change (straight forwarding of struts and cells) and failure of the struts. In order to confirm the increase in Young's modulus due to the morphology change, microsamples with several struts and nodes were taken out by cutting off from the foams (whose morphology will be shown later in Figs. 2 and 8), and the change of Young's modulus with applied strain was measured from the slope of the elastic deformation region in the load-strain curve during unloading, as similarly as in the foam samples. In the tensile test, the gage length was taken to be $1 \mathrm{~mm}$. The end regions of the micro-sample were glued to a $100 \mu \mathrm{m}$ thick paper with an epoxy resin (High Super 30, Cemedine Co., Ltd., Tokyo, Japan), as shown in Fig. 1(a). After gripping the test sample in the tensile machine, the paper was cut so that the applied load was carried only by the micro-sample (Fig. 1(b)). The tensile test was carried out at room temperature with a universal tensile testing machine (MMT-10N-2, Shimadzu Co., Kyoto, Japan), which is designed for the measurement of strength of small diameter $(5-20 \mu \mathrm{m})$ fibers and has been used for estimation of strength of $\mathrm{SiC}$ fiber ${ }^{15)}$ and $\mathrm{Nb}_{3} \mathrm{Al}$ superconducting filaments. ${ }^{16)}$ The deformation and failure behavior was observed with a digital microscope (KEYENCE VHX500SP1639).

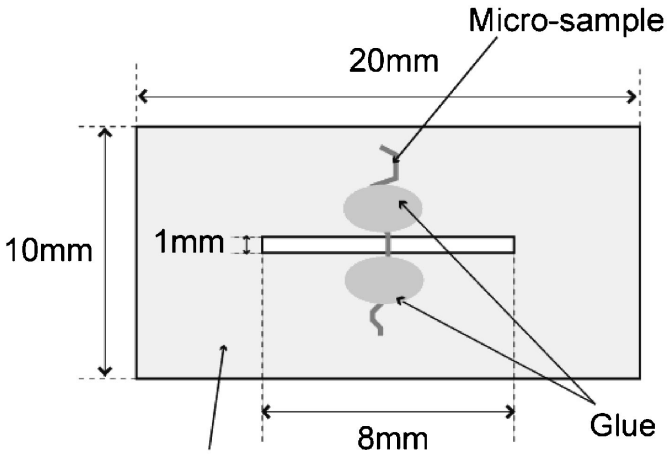

Paper tab

(a)

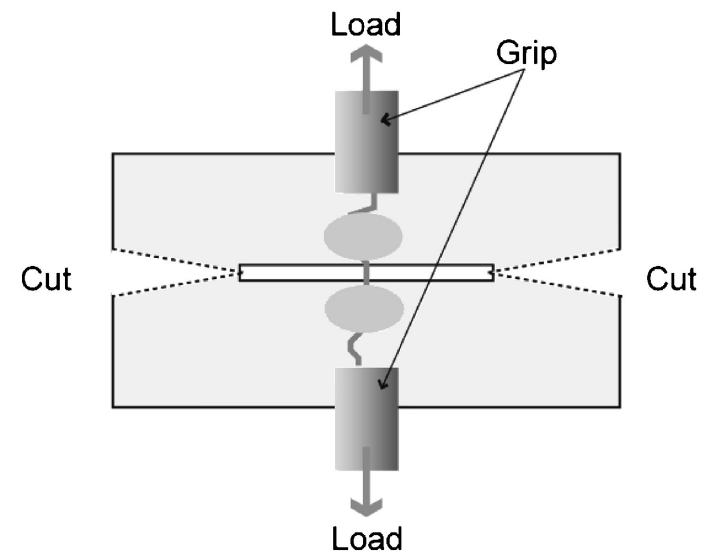

(b)

Fig. 1 Tensile test method of the micro-samples taken out of the foam.

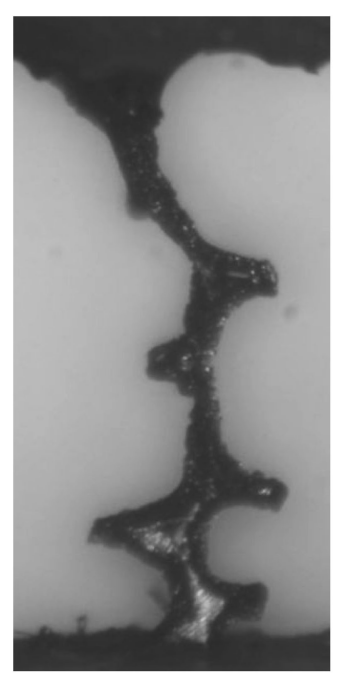

(a)

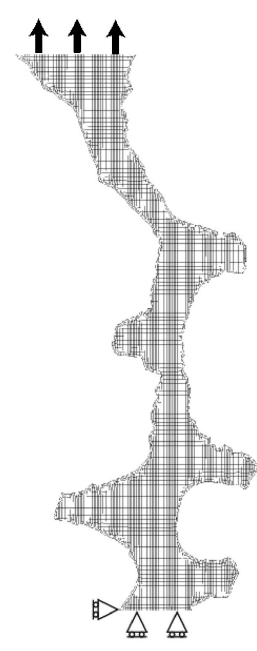

(a')

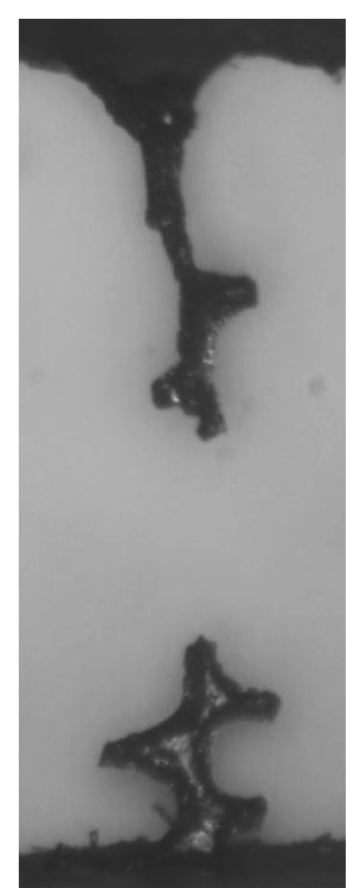

(b)

Fig. 2 Modeling of the micro-sample for FEM analysis to examine the influence of the morphology change on Young's modulus. (a) Micro-sample taken out of the nickel foam. (a') FEM model using the configuration of the micro-sample shown in (a). (b) Failure morphology of the micro-sample which was straight-forwarded toward the tensile axis by plastic deformation. 
For numerical demonstration of the increase in Young's modulus due to the change of the morphology of cells and struts, a finite element analysis was conducted. Figure 2 shows the micro-sample used for modeling. (a) shows the original shape of the micro-sample taken out of the nickel foam. (b) shows the failure morphology of the micro-sample which was straight-forwarded toward the tensile axis by plastic deformation. The influence of the straight-forwarding toward the tensile axis of the sample on the Young's modulus value was calculated using the FEM model shown in $\left(a^{\prime}\right)$, which was taken from the configuration of the micro-sample shown in (a).

The stress analysis was carried out using the commercial finite element code MARC/Mentat ${ }^{\mathrm{TM}}$ under the plane stress condition. The yielding condition for the metal was given by the von Mises criterion. As the mechanical properties of the nickel in the present foam were unknown, the following values reported for full annealed polycrystalline nickel ${ }^{17}$ ) were used: Young's modulus $E_{\mathrm{Ni}}=207 \mathrm{GPa}$, Poisson's ratio $=0.3$, tensile strength $\left(\sigma_{\mathrm{UTS}}\right)=317 \mathrm{MPa}$, yield stress $\left(\sigma_{\mathrm{Y}}\right)=59 \mathrm{MPa}$ and nominal strain at ultimate stress $\left(e_{\mathrm{UTS}}\right)=30 \%$. The true stress $\left(\sigma_{\mathrm{t}}\right)$-true strain $(\varepsilon)$ curve was expressed with the Ludwik's equation given by

$$
\sigma_{\mathrm{t}}=\sigma_{\mathrm{y}}+K\left(\varepsilon-\varepsilon_{\mathrm{y}}\right)^{n}
$$

Here $\varepsilon_{\mathrm{y}}$ is the yield strain, and $K$ and $n$ are the constants. Using the well known expressions in solid mechanics, we have $\varepsilon_{\mathrm{y}}=\sigma_{\mathrm{Y}} / E_{\mathrm{Ni}}$ (Hooke's law), $\varepsilon=\ln (1+e)$ (relation of true strain $(\varepsilon)$ to nominal one $(e)), \sigma_{\mathrm{UTS}}=\left\{\sigma_{\mathrm{y}}+K\left(\varepsilon_{\mathrm{UTS}}-\right.\right.$ $\left.\left.\varepsilon_{\mathrm{y}}\right)^{n}\right\} \exp \left(-\varepsilon_{\mathrm{UTS}}\right)$ (relation of nominal tensile strength $\left(\sigma_{\mathrm{UTS}}\right)$ to true strain $\left(\varepsilon_{\mathrm{UTS}}\right)$ at ultimate stress), and $\left[\mathrm{d}\left\{\sigma_{\mathrm{t}} \exp (-\varepsilon)\right\} /\right.$ $\mathrm{d} \varepsilon](\mathrm{d} \varepsilon / \mathrm{de})=0$ at $e=e_{\mathrm{TUS}}$ (necking condition). From these relations with the reported mechanical property values, the $\varepsilon_{\mathrm{y}}, n$ and $K$ in eq. (1) were calculated to be $2.85 \times 10^{-4}$, 0.262 and $502 \mathrm{MPa}$, respectively.

\section{Results and Discussion}

\subsection{Change of Young's modulus of the foams with increasing tensile strain}

Figure 3 shows the nominal stress-nominal strain curves of the (a) nickel and (b) copper foams under repeated loading-unloading-reloading, together with the deformation and failure stages (I characterized by the elastic deformation, II by plastic deformation, III by the co-occurrence of plastic deformation and cumulative failure of struts and IV by the successive failure of the struts by the chain reaction of strut failure nearly in a limited cross-section ${ }^{14)}$ ). The stress increased, reaching the ultimate stress, and then decreased significantly with increasing applied strain. The strains at ultimate stress indicated by the broken lines were 5.2 and $6.2 \%$ for the tested nickel and copper foams, respectively. Details of the stress-strain behavior in the unloadingreloading process in the portions noted as Fig. 4(a) and (b) in Fig. 3(b) are presented at high magnification in Fig. 4(a) and (b), respectively. The hysteresis is clearly detected by the present method using the non-contact extensometer. Upon unloading and also upon re-loading, the foam deforms elastically. The slope in the elastic region in the stress-strain curve, corresponding to Young's modulus, is shown with
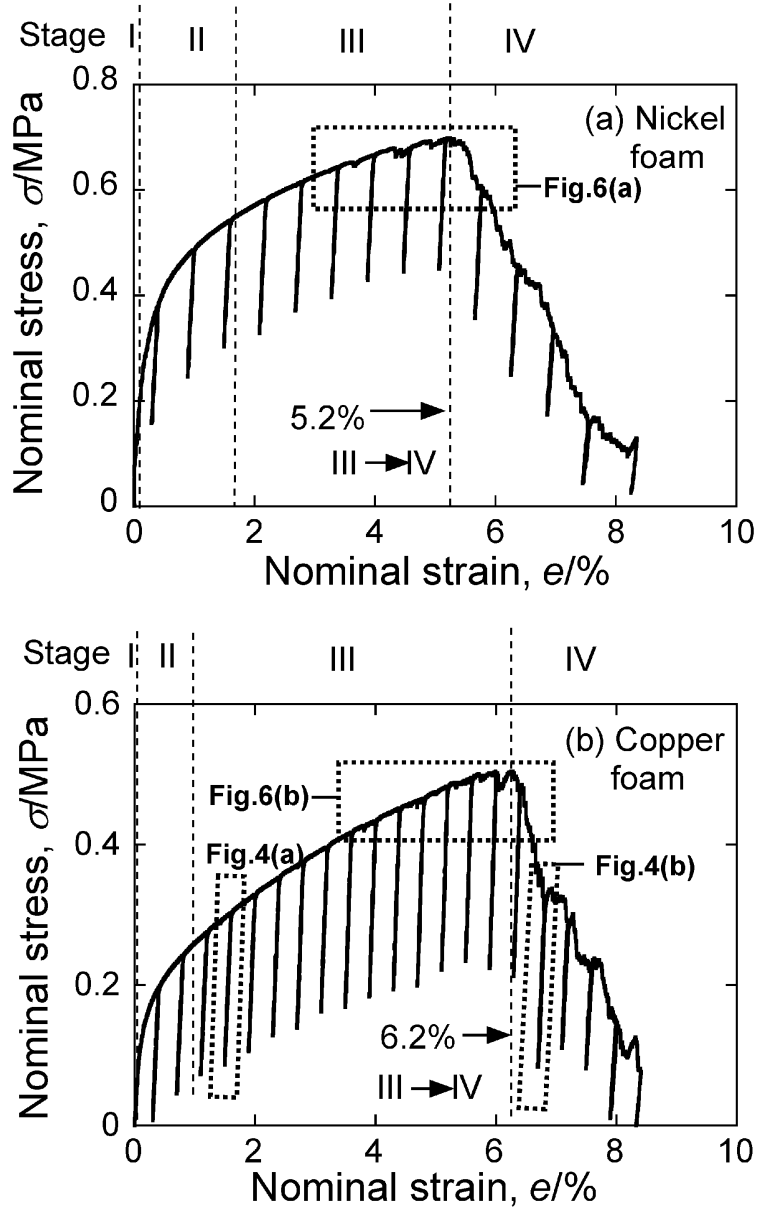

Fig. 3 Measured stress-strain curves of the (a) nickel and (b) copper foams under repeated loading-unloading-reloading. The details of the stressstrain behavior in the unloading-reloading process in the portions indicated by Fig. 4(a) and Fig. 4(b) will be presented later in Fig. 4. Details of the stress-strain behavior with serrations indicated by Fig. 6(a) and Fig. 6(b) will be presented later in Fig. 6.

broken lines. The deviation from the broken lines stems from the plastic deformation. In the present work, the Young's modulus values were estimated from the slope of the elastic region in the unloading process.

Figure 5 shows the variation of the Young's modulus of (a) the nickel and (b) copper foams with increasing applied nominal strain $e$. The broken lines show the strains at ultimate stress mentioned above. The change of electric resistance measured in our preceding work $^{14)}$ is also presented for reference. The following features are read from Fig. 5. (1) The Young's modulus increases, reaching maximum, and decreases in both foams. (2) The Young's modulus is reduced seriously in accordance with the increase in electric resistance beyond the strain at ultimate loading indicated by the broken lines. The reason why these features are found is discussed below from the viewpoint of deformation and failure behavior of the foams.

\subsection{Relation of the deformation and failure behavior to change of Young's modulus value \\ 3.2.1 Morphology-change of the cells and struts}

The cells and struts are elongated and are straight forwarded in the tensile direction. ${ }^{14)}$ It is emphasized that 

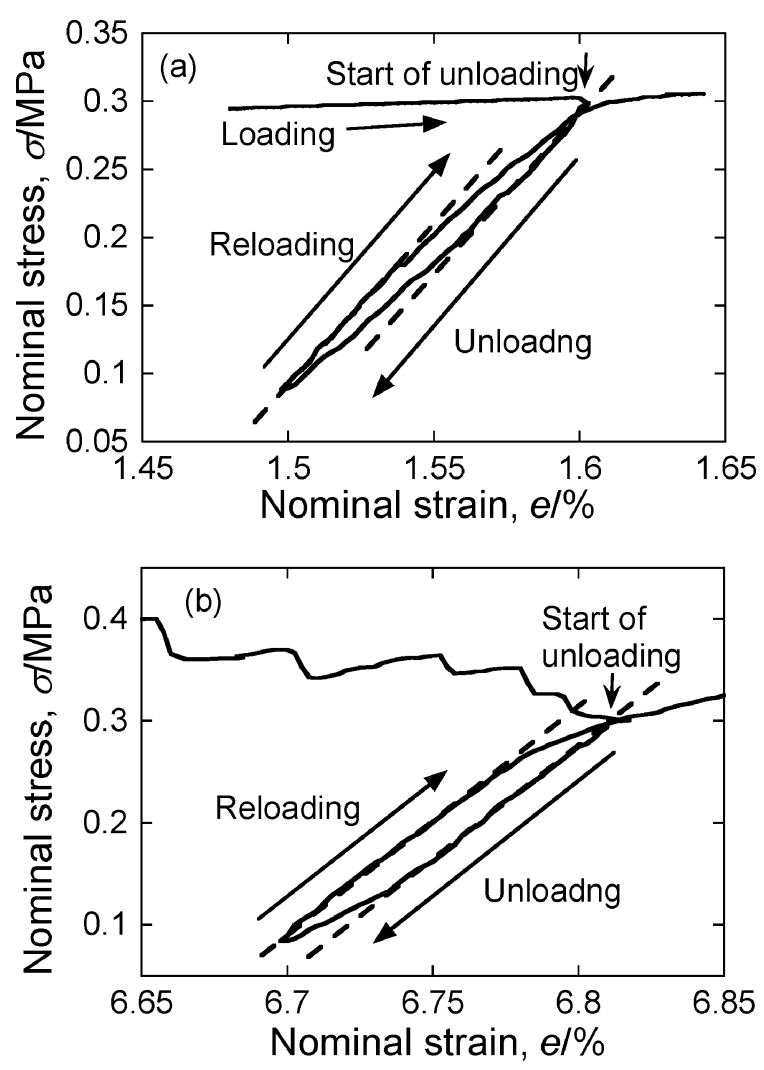

Fig. 4 Detailed stress-strain behavior of the copper foam in the unloadingreloading process. (a) and (b) show the behavior of the portions indicated by Fig. 4(a) and Fig. 4(b) in Fig. 3(b), respectively.

the shape change is caused by the plastic deformation and once it takes place, it is eternal. Accordingly it is expected that, as the curved cells and struts are straight forwarded, accompanied by the plastic deformation, the Young's modulus becomes higher. Andrews et al. ${ }^{18)}$ and Simone and Gibson $^{19)}$ studied the influence of the curvature and corrugation of struts on the mechanical properties of foams. Their calculation results showed that the existent cell wall curvature and corrugations act to reduce the stiffness of aluminum foams. Their results, in turn, suggest that, when the cell and struts are straight forwarded in the stress direction, the Young's modulus increases. Zhou et al. ${ }^{20)}$ conducted micro-scale tensile test on individual struts taken out of the open cell aluminum foams. They found that the strut becomes parallel to the loading axis under the applied load. Their results indicate that the Young's modulus of the foam can be higher with increasing applied strain due to the shape change.

The present and the reported results show that the Young's modulus increases due to the morphology change, as similarly as the slope of the stress-strain curve increases with increasing applied strain when loose strands are stretched. To confirm the effect of the morphology change on the increase in Young's modulus, further experiment and analysis were carried out using the micro-samples taken out of the foams. Details of the results will be presented later in 3.3 .

\subsubsection{Failure of struts}

If only the morphology change occurs, the Young's modulus is expected to increase with increasing applied strain. However, as shown in Fig. 5, the Young's modulus
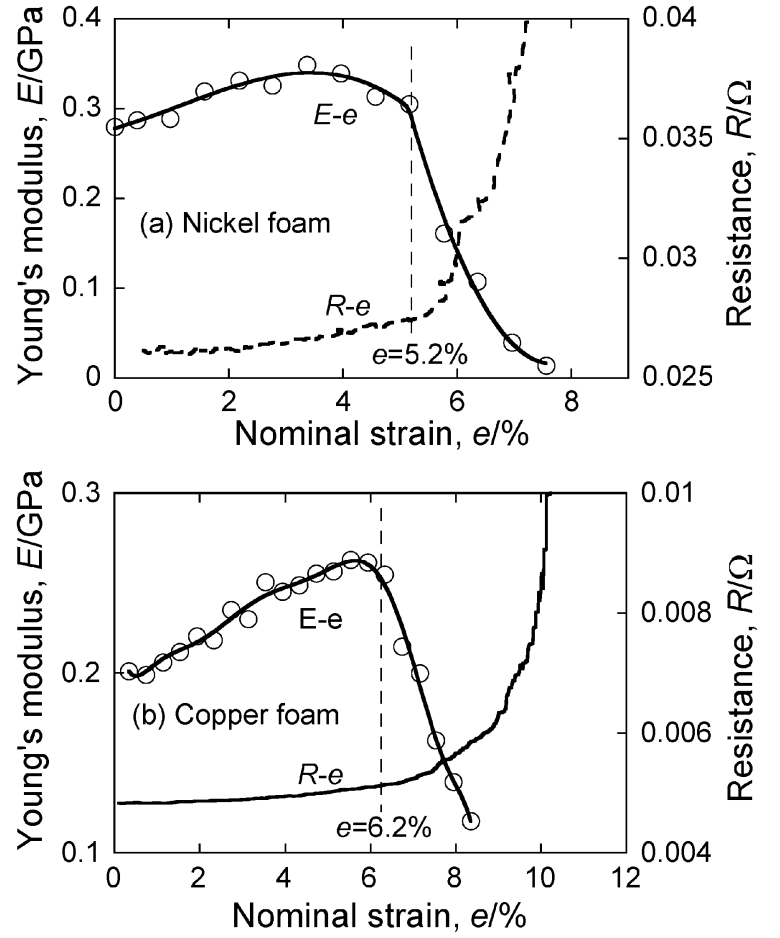

Fig. 5 Variations of the Young's modulus and electrical resistance of (a) the nickel and (b) copper foams with increasing applied strain $e$.

value decreased at high applied strains beyond the strain at ultimate stress. As the reason for the decrease, the failure of struts is mentioned.

In Fig. 3, the stress increased up to the ultimate stress and then decreased beyond $e=5.2$ and $6.2 \%$ in the tested nickel and copper foams, respectively. The portions at nominal strain $e=3-6 \%$ and $3.5-7 \%$ for nickel and copper foams are presented at high magnification in Fig. 6(a) and (b), respectively. In both foams, the serrations took place due to the failure of the struts. In the preceding work, ${ }^{14)}$ it was found that the struts are failed cumulatively in stage III up to the ultimate stress at $e=5.2$ and $6.2 \%$ for the nickel and copper foams, respectively, and then they are failed successively in stage IV nearly in a limited cross-section within around the cell size in longitudinal direction, due to an enhanced strain concentration.

Even in stage III, the number of failed struts increases, which acts to reduce the stress carrying capacity and therefore acts to reduce Young's modulus value in comparison with the expected value under no failure. In stage IV, the successive failure occurs nearly in a limited cross-section, resulting in serious reduction. In stage IV, the effect of the shape change to raise the Young's modulus is overcome by the effect of the strut failure to reduce the Young's modulus. Accordingly, due to the competition of the positive effect (morphology change), which increases with increasing applied strain, and the negative one (struts failure), which increases also with increasing applied strain, the Young's modulus increases, reaching maximum, and decreases with increasing strain in both foams. The strain-dependence of the Young's modulus, which is not found in bulk materials, is a feature of the metallic foams especially with high porosity as in the present foams. 
$5.2 \%$

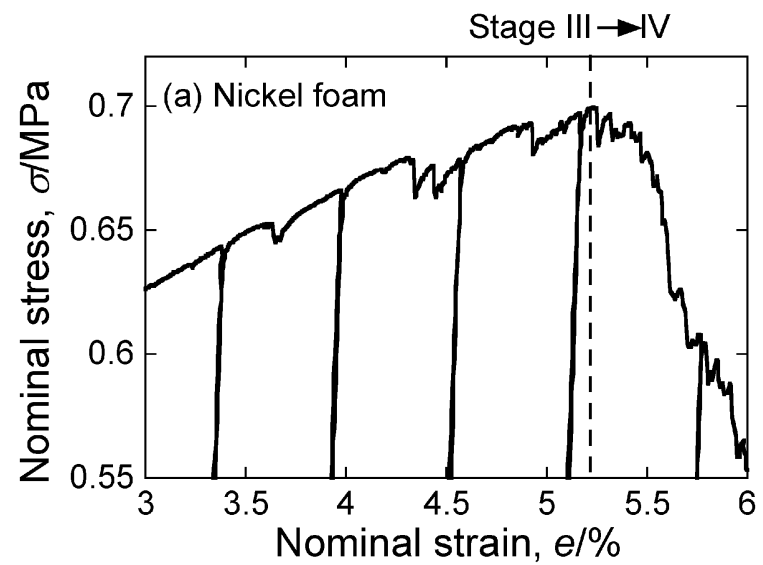

$6.2 \%$

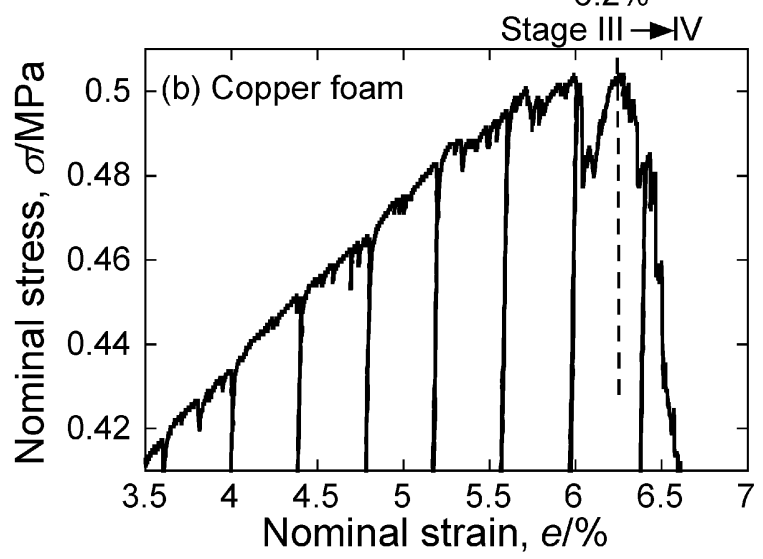

Fig. 6 Nominal stress-nominal strain curves in the stages III and IV of (a) the nickel foam, taken from the portion indicated by Fig. 6(a) in Fig. 3(a), and of (b) the copper foam, taken from the portion indicated by Fig. 6(b) in Fig. 3(b). Both foams show serrations due to the failure of struts.

\subsection{Effects of morphology change of struts on Young's} modulus

As shown above, the change of the Young's modulus of foams with increasing applied strain is attributed to the morphology change (straight forwarding of struts and cells) and failure of the struts. The increase in Young's modulus due to the morphology change was confirmed by the additional tests and calculation for micro-samples, as follows.

Examples of load-apparent strain $\left(e_{\text {app }}\right)$ curve of the microsamples taken out of nickel foams are shown in Fig. 7. The shape of the curve was different among the samples. The configuration of sample A in Fig. 7 was rather straight along the tensile axis as in Fig. 2. In this case, the stress went up from the initial stage, similarly to that of bulk wire. On the other hand, when the sample configuration was more curved as in Fig. 8 (details will be show below), the load-strain curve

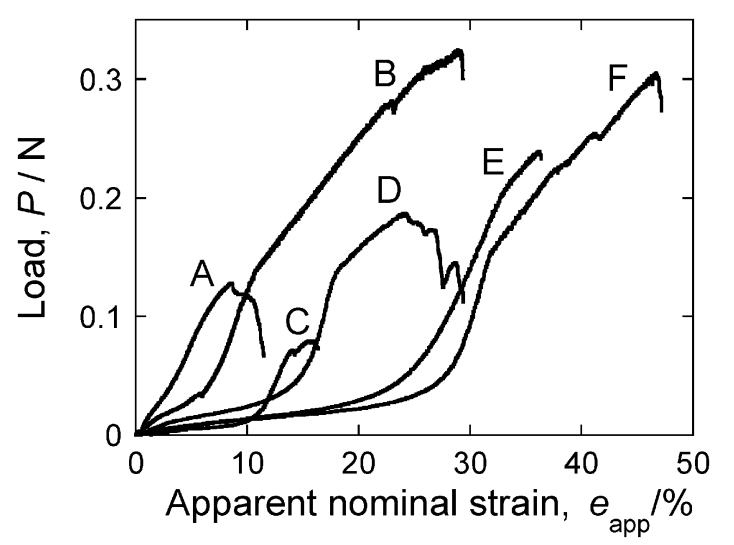

Fig. 7 Examples of the load-apparent strain curve of micro-sample taken out of the nickel foam.

\section{Tensile direction}

\section{Before tensile test}

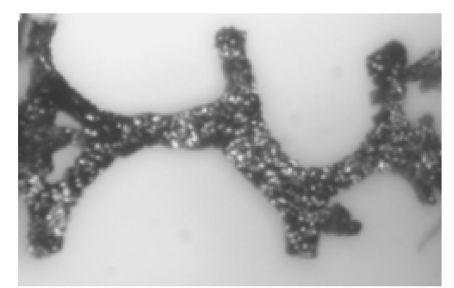

(a)

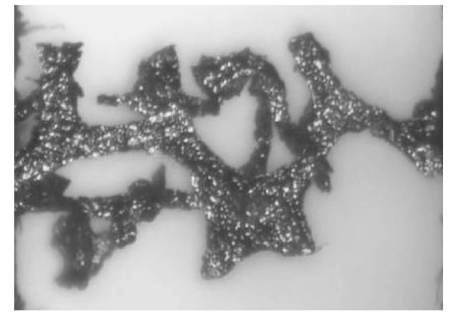

(b)
After tensile test

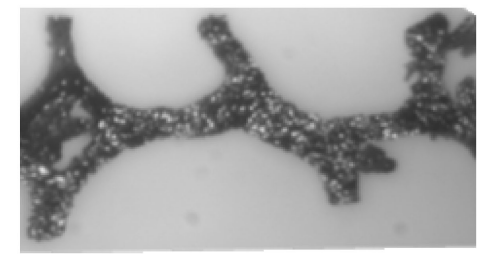

(a')

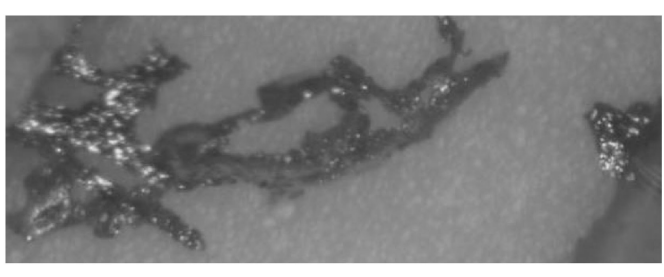

(b') $\quad 500 \mu \mathrm{m}$

Fig. 8 Digital microscope image of $((a),(b))$ the micro-samples taken out from the copper foam, together with $\left(\left(\mathrm{a}^{\prime}\right),\left(\mathrm{b}^{\prime}\right)\right)$ the stretched shape after tensile test for comparison. 
turned to S-shape type (samples B to F), similarly to that of loose strands.

Figure 8 shows the digital microscope image of ((a), (b)) the micro-samples taken out from the copper foam, together with $\left(\left(a^{\prime}\right),\left(b^{\prime}\right)\right)$ the stretched shape after tensile test for comparison. These samples were not straight and had curvatures in original state. After the tensile test, the samples were straight forwarded along the tensile direction. Figure 9(a) shows the load-strain curves with repeated loadingunloading-reloading treatment for the samples (a) and (b). As the configuration and cross-sectional area varied with the sample length, the definite cross-sectional area could not be defined, due to which just the load was used instead of stress. As the non-contact extensometer could not be used for the test of micro-samples, the displacement of the cross-head was used for strain estimation. Accordingly, the estimated strain was apparent (noted as $e_{\text {app }}$ in Fig. 9(a)), containing the strain of the sample itself $e_{\mathrm{S}}$ and the strain of the system $e_{\mathrm{M}}$ for measurement (sum of the strain of the machine system and the strain of the paper with glue (Fig. 1)). In the present work, the following correction was made to diminish the influence of the latter stain by estimation of the compliance of the measurement system using the carbon fiber (GRANOC XN-35 with a nominal Young's modulus $E_{\mathrm{f}}=340 \mathrm{GPa},{ }^{21)}$ Nippon Graphite Fiber Corp.) The cross-sectional area $A_{\mathrm{f}}$ was measured to be $93 \mu \mathrm{m}^{2}$ on average.

Noting the sample length before tensile test as $L_{0}$ and the displacement of the sample itself as $\Delta L_{\mathrm{S}}$ under the load $P$, the relation among $E_{\mathrm{f}}, A_{\mathrm{f}}, \Delta L_{\mathrm{S}}, L_{0}$ and $P$ is expressed by

$$
P=E_{\mathrm{f}} A_{\mathrm{f}} \Delta L_{\mathrm{S}} / L_{0}
$$

Noting the displacement of the measurement system as $\Delta L_{\mathrm{M}}$ under the applied load $P$, the compliance $C_{\mathrm{M}}$ of the measurement system is expressed by $C_{\mathrm{M}}=\Delta L_{\mathrm{M}} / P$. As the total apparent displacement $\Delta L_{\text {app }}$ is given by $\Delta L_{\text {app }}=$ $\Delta L_{\mathrm{s}}+\Delta L_{\mathrm{M}}$, we have

$$
\Delta L_{\mathrm{app}} / P=L_{0} /\left(E_{\mathrm{f}} A_{\mathrm{f}}\right)+C_{\mathrm{M}}
$$

In the experiment, we took $L_{0}=10,20,30,40$ and $50 \mathrm{~mm}$, and conducted tensile test for $10 \sim 15$ carbon fiber specimens for each $L_{0}$. The plot of $\Delta L_{\text {app }} / P$ against $L_{0}$ is presented in Fig. 9(b). By application of the least square method to the linear relation between $\Delta L_{\mathrm{app}} / P$ and $L_{0}$, we had the slope $1 /\left(E_{\mathrm{f}} A_{\mathrm{f}}\right)=0.029 \mathrm{~N}^{-1}$. Substituting $A_{\mathrm{f}}=93 \mu \mathrm{m}^{2}$ into the obtained slope value, we had $E_{\mathrm{f}}=370 \mathrm{GPa}$. This value was close to $340 \mathrm{GPa}$, indicating that the present method gave satisfactory result without serious loss of accuracy. The $C_{\mathrm{M}}$ value was estimated to be $28 \mu \mathrm{m} / \mathrm{N}$ from the extrapolation. These results mean that the displacement $L_{\mathrm{M}}$ of the measurement system is given by $P C_{\mathrm{M}}$ and the strain of $P C_{\mathrm{M}} / L_{0}$ is added in the strain $e_{\text {app }}$. Thus the strain of the sample $e_{\mathrm{S}}$ is given by $e_{\mathrm{S}}=e_{\mathrm{app}}-P C_{\mathrm{M}} / L_{0}$. The initially obtained $P-e_{\text {app }}$ curve was converted to $P-e_{\mathrm{S}}$ curve, from which the overall Young's modulus $E_{\text {overall }}$ (slope in the elastic region in the $P-e_{\mathrm{S}}$ curve during unloading) was obtained.

Thus obtained $E_{\text {overall }}$ values plotted against the corrected strain $e_{\mathrm{S}}$ are shown in Fig. 9(c). The $E_{\text {overall }}$ values, read directly from the slope of the load-strain curve in unloading process, increased from $6.0 \mathrm{~N}$ at $e_{\text {app }}=7.1 \%$ to $15 \mathrm{~N}$ at $e_{\text {app }}=12.5 \%$ in sample (a) and from $4.4 \mathrm{~N}$ at $e_{\text {app }}=18.8 \%$
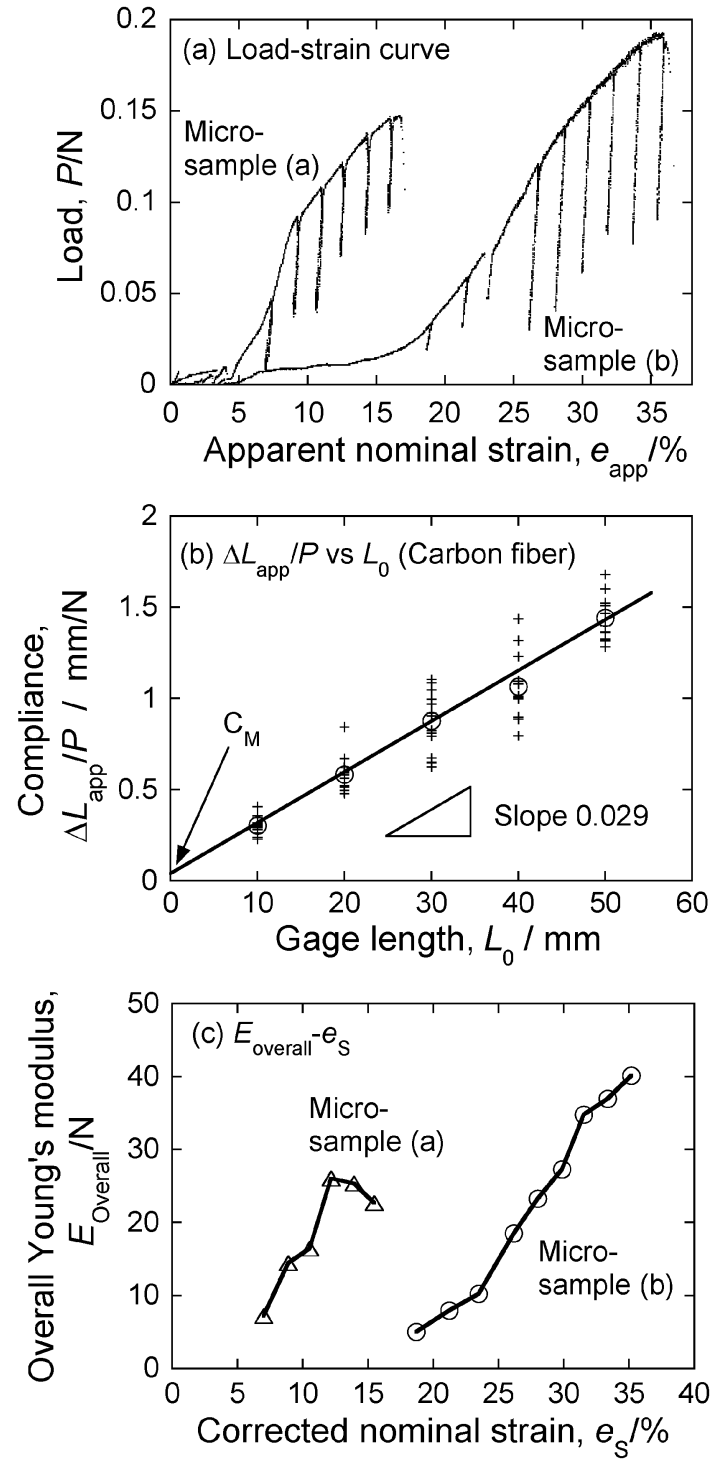

Fig. 9 (a) Measured load-apparent strain curves with repeated loadingunloading-reloading treatment of the micro-samples taken out of the copper foam, (b) plot of the compliance against sample length, measured using carbon fiber to estimate the compliance of the measurement system $C_{\mathrm{M}}$ and (c) change of the overall Young's modulus with applied strain of the micro-samples, corrected with the compliance $C_{\mathrm{M}}$.

to $18.9 \mathrm{~N}$ at $e_{\text {app }}=35.7 \%$ in sample (b). Even without correction, significant increase in $E_{\text {overall }}$ with strain was clearly detected. After the correction, the $E_{\text {overall }}$ values increased from $7.2 \mathrm{~N}$ at $e_{\mathrm{S}}=7.0 \%\left(e_{\mathrm{app}}=7.1 \%\right)$ to $26 \mathrm{~N}$ at $e_{\mathrm{S}}=12.2 \%\left(e_{\mathrm{app}}=12.5 \%\right)$ in sample (a) and from $5.0 \mathrm{~N}$ at $e_{\mathrm{S}}=18.7 \%\left(e_{\mathrm{app}}=18.8 \%\right)$ to $40.1 \mathrm{~N}$ at $e_{\mathrm{S}}=35.2 \%\left(e_{\mathrm{app}}=\right.$ $35.7 \%$ ) in sample (b). As a result, more sharp increase in $E_{\text {overall }}$ could be revealed by the present correction.

The following interesting indications were obtained from Fig. 9(c).

(1) As shown in Fig. 8, the morphology of the samples was changed by applied strain. The samples with curved and complex configuration were stretched toward the direction parallel to the tensile stress. According to such a morphology change of samples, the $E_{\text {overall }}$ values increased largely. This result demonstrates that the morphology change acts to raise Young's modulus in the foams. 


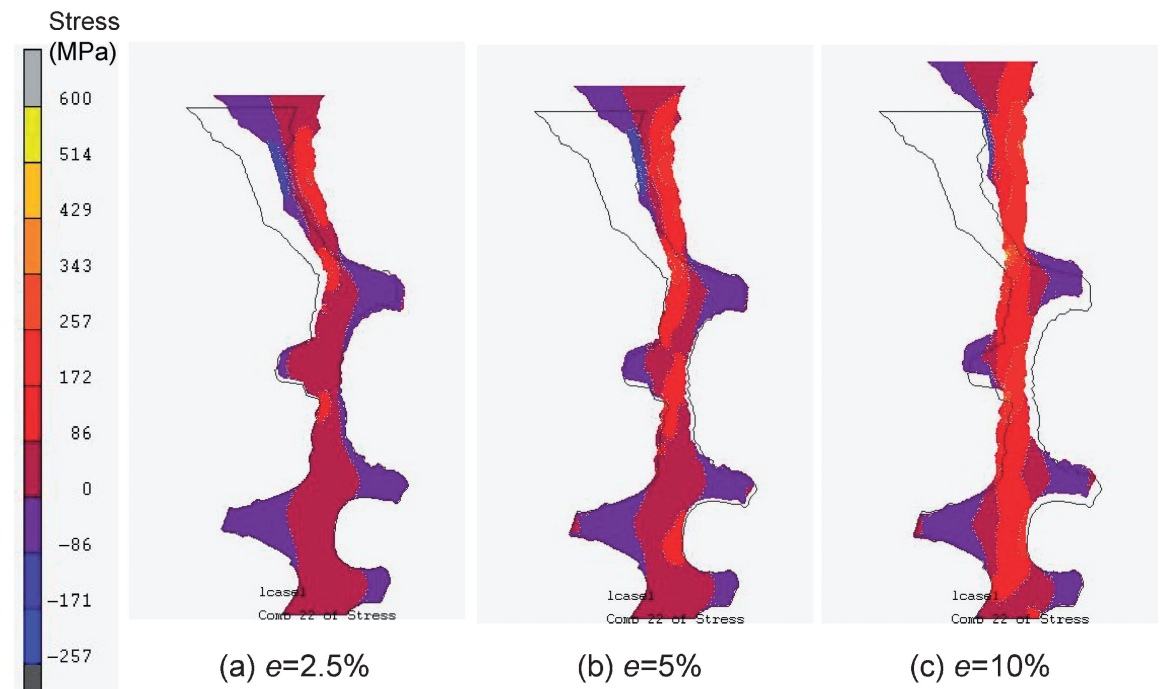

Fig. 10 Simulated change of the shape and true stress distribution with increasing applied nominal strain in the model micro-sample (Fig. 2(a) and $\left.\left(a^{\prime}\right)\right)$. The original shape is shown with the black solid curves.

(2) The $E_{\text {overall }}$ values of samples (a) and (b) increased around by several times in accordance with the increase in applied strain. In the experimental result of the foams, the increase in the Young's modulus from the initial value at $e=0 \%$ was, however, $25-30 \%$ at $e=5 \sim 6 \%$ (Fig. 5). The following two factors could be mentioned as the reason why the increase in Young's modulus of the foams is small in comparison with that of micro-samples. (A) Among the struts, some struts, which are originally rather straight such as sample A in Fig. 7, contribute to Young's modus of foams even at $e=5 \sim 6 \%$. However, the curved struts with S-shape load-strain curves (samples B to E in Fig. 7) do not contribute effectively since the strain of $5 \sim 6 \%$ is not sufficient for them to raise Young's modulus. Namely there coexist effective and ineffective struts and only the effective struts contribute to Young's modulus of foams at $e=5 \sim 6 \%$. (B) It is noted again that, though the number of failed struts at $e=5 \sim 6 \%$ is small in comparison with the total number of struts, the failure of struts enhances the strain concentration on the neighboring struts and causes successive failure nearly in a limited cross-section beyond $e=5 \sim 6 \% .{ }^{14)}$ Such a successive failure reduces seriously the cross-sectional area of ligament in the failing cross-section and therefore the stress carrying capacity of foams. Accordingly, even though most struts can exhibit high Young's modulus when stretched up to higher strain, the struts in the failing cross-section cannot contribute to the increase in Young's modulus of foams due to the more reduced ligament cross-sectional area. Also as the stress level is reduced in comparison with that at $e=5 \sim 6 \%$, the struts outside the failing cross-sectional area cannot be strained more than 5 6\%. Therefore, they cannot exhibit high Young's modulus. In this way, the failure of struts acts as a trigger for the suppression of increase in Young's modulus of foams.

As shown above, the increase in Young's modulus is attributed to the change of the shape of cells and struts. In order to reconfirm the effect of the morphology change on the increase in Young's modulus numerically, a simulation was carried out with the finite element calculation, whose model has been shown in Fig. 2. Figure 10 shows the simulated change of the morphology and true stress distribution with increasing applied nominal strain of the model micro-sample. In these figures, the true stress in the tensile direction is shown. Initial shape of the model is shown with black curves for comparison.

With increasing applied strain, the longitudinal strut-parts elongate more along the tensile direction. Accordingly, the micro-sample tends to be straight forwarded along the tensile axis as clearly shown by the difference in shape after deformation from the original shape.

Figure 11 shows the simulated (a) load-nominal strain curve of the model micro-sample with repeated loadingunloading-reloading process and (b) change of overall Young's modulus $E_{\text {overall }}$ with increasing applied strain $e$. As the curvature of the model (Fig. 2) was not large in comparison with that of the samples shown in Fig. 8, the stress went up from the initial stage in the load-strain curve as similarly as sample A in Fig. 7. In accordance with the change of the straight forwarded-shape change (Fig. 10), the $E_{\text {overall }}$ increased greatly, as similarly as the experimental results in Fig. 9(c). The present simulation, together with the experimental results, demonstrates the great increase in Young's modulus due to the morphology change.

\section{Conclusions}

The change of Young's modulus with increasing applied tensile strain in the open cell nickel and copper foams, fabricated by the slurry foaming process, as well as the micro-samples taken out of foams was investigated experimentally and analytically. The main results are summarized as follows.

(1) The feature of the change of the Young's modulus particular to the metal foams with high porosity was revealed; the Young's modulus increases, reaching maximum and then decreases with increasing applied strain. 

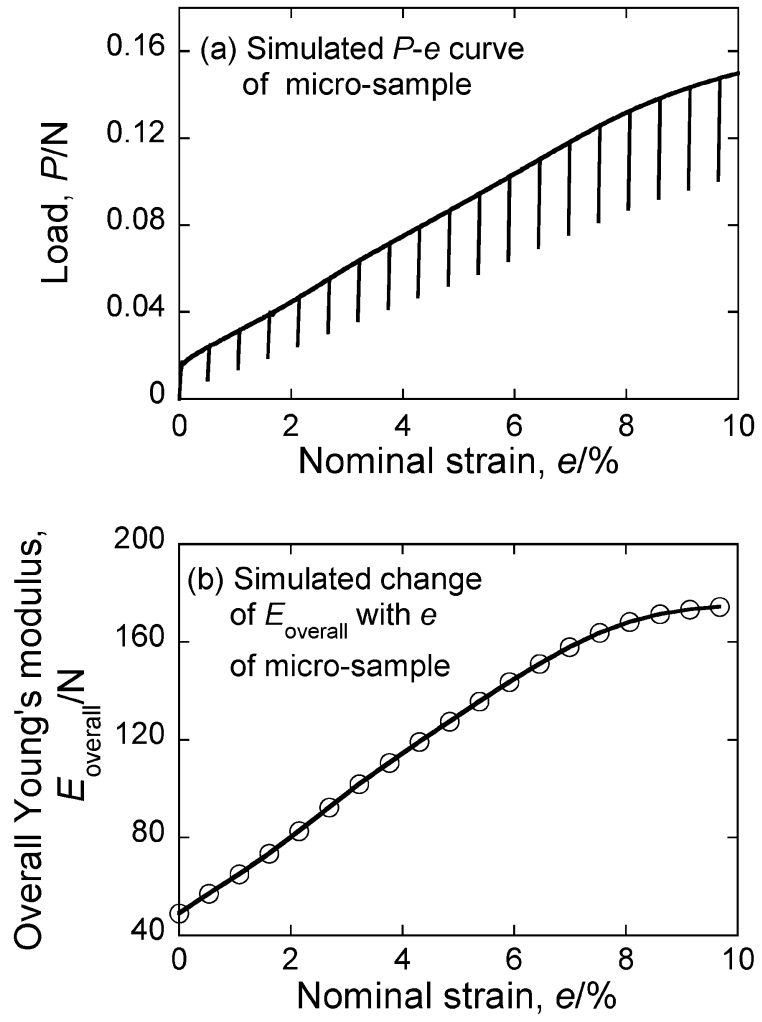

Fig. 11 Simulated (a) load-nominal strain curve with repeated loadingunloading-reloading process of the model micro-sample (Fig. 2(a) and $\left(a^{\prime}\right)$ ) and (b) change of overall Young's modulus with increasing applied nominal strain.

(2) Two competing factors affected on the Young' modulus value. One factor was the change of morphology of struts and cells arising from the applied strain-induced plastic deformation, according to which the struts and cells were elongated and were straight forwarded in the tensile direction. This factor acted to raise the Young's modulus with increasing applied strain. Another was the failure of struts. This factor acted to reduce the Young's modulus with increasing applied strain. Accordingly, due to the competi- tion of the former and latter factors, the Young's modulus increased, reaching maximum and then decreased with increasing applied strain in both foams.

(3) The increase in Young's modulus with increasing applied strain due to the morphology change of struts was confirmed by the additional test of micro-samples composed of several struts and nodes and by the simulation based on the finite element analysis using a model micro-sample.

\section{REFERENCES}

1) D. Leitlmeirr, H. P. Degischer and H. J. Flankl: Adv. Eng. Mater. 4 (2002) 735-740.

2) J. Banhart: J. Mater. 52 (2000) 22-27.

3) H. Nakajima: Prog. Mater. Sci. 52 (2007) 1091-1173.

4) H. Nakajima, M. Tane, S. Hyun and S. Suzuki: Materia Japan 47 (2008) 196-202.

5) M. Tane and H. Nakajima: Mater. Trans. 47 (2006) 2183-2187.

6) F. Baumgärtner, I. Duarte and J. Banhart: J. Adv Eng. Mater. 2 (2000) $168-174$.

7) M. Kobashi and N. Kanetake: Materia Japan 47 (2008) 178-181.

8) N. Kanetake, M. Kobashi and S. Tsuda: MetFoam 2007-Proc. 5th Int. Conf. on Porous Metals and Metallic Foams, (Destech Publications, Toronto, 2008) pp. 63-66.

9) M. Wada: Chem. Chem. Industry 54 (2001) 811-813.

10) H. N. D. Wadley: Adv. Eng. Mater. 14 (2002) 726-733.

11) H. Nakajima and T. Ide: Metal. Mater. Trans. A 39 (2008) 390-394.

12) M. Kobashi, K. Kuze and N. Kanetake: Adv. Eng. Mater. 9 (2006) 836840 .

13) N. Kanetake and M. Kobashi: J. High Temp. Soc. 34 (2008) 45-50.

14) S. Ochiai, S. Nakano, Y. Fukazawa, M. S. Aly, H. Okuda, K. Kato, T. Isobe, K. Kita and K. Honma: Mater. Trans. 51 (2010) 699-706.

15) K. Morishita, S. Ochiai, H. Okuda, T. Ishikawa, M. Sato and T. Inoue: J. Am. Ceram. Soc. 89 (2006) 2571-2576.

16) S. Ochiai, T. Sawada, S. Nishino and M. Hojo: Intermetallics 4 (1996) S201-S208.

17) Online Materials Information Resource-MatWeb. http://www.matweb. com/search/DataSheet for annealed nickel

18) E. Andrews, W. Sanders and L. J. Gibson: Mater. Sci. Eng. A 270 (1999) 113-124.

19) A. Simone and L. J. Gibson: Acta Mater. 46 (1998) 3929-3935.

20) J. Zhou, S. Allameh and W. O. Soboyejo: J. Mater. Sci. 40 (2005) 429439.

21) Nippon Graphite Fiber Corp.: Catalogue "Pitch based carbon fiber GRANOC”. 